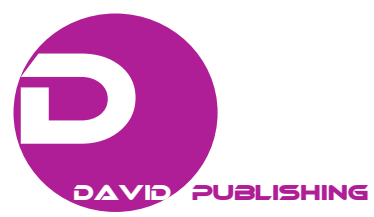

\title{
Magnetic Effects in Brain Chemistry
}

\author{
Aibassov Yerkin", Yemelyanova Valentina, Tussupbayev Nessipbay, Shakieva Tatyana, Yerzhanova Zhadyra, \\ Bulenbayev Maxat and Blagikh Evgeniy \\ Research Institute of New Chemical Technologies and Materials, Kazakh National University Al-Farabi, Almaty 005012, Kazakhstan
}

\begin{abstract}
Chemistry of the human brain has two components - the basic chemistry common to all mammals and chemistry of thinking inherent to man. The authors proposed a mechanism of induction and thermodynamic features of the brain. The authors offered the mechanisms of the work RAM (physical) and permanent (chemical), the brain's memory, including the model of nonlocal quantum correlations.
\end{abstract}

Key words: Brain, isomerism, benzidine rearrangement, magnetic field.

\section{Introduction}

In recent years, many new works, are devoted to the chemistry of the brain. The basic structural and functional unit of the brain is the nerve cell. It generates and conducts electrical impulses - the ROP (Redox Potential). ROP associated with the movement of the charges induces a local vortex electromagnetic field. The metric principle and the velocity of the photon will be determined by physical properties and structural features of a neuron and its environment. This is necessary for energy-generating and mechanism to provide impulses and metabolites responsible for the synthesis of the cell body, dendrites and its nucleus. Axons in symbiosis with glia (oligodendrocytes, astrocytes) broadcast metabolites and impulses, realizing their energy and information through the synthesis and action of neurotransmitters at synapses. The capacity of the nerve cell provides the energy oxidation of glucose, which is transformed into the energy of the mitochondria-energy bonds of ATP (adenosine triphosphate). The nerve cells of the energy of ATP are converted into energy quasiphotons, into chemical energy synthesized substances, metabolites and the

*Corresponding author: Aibassov Erkin Zhakenovich, professor, research fields: metalorganic chemistry of uranium and thorium, $\mathrm{As}, \mathrm{Sb}$ and $\mathrm{Bi}$. kinetic energy of the molecules of the medium (the heat). Due to the same energy are axonal growth, neural development and neuroglial communications networks, which, in particular, are responsible for the mechanical integrity of the cytoskeleton brain [1-11].

Thus, the behavior of the brain as a single physical system in the first place is subject to the laws of classical thermodynamics and electrophysics. Within the framework of these laws made the metabolism of the neuron and the brain performs its basic functions controlling homeostasis and timely launch of the mechanism of sexual reproduction. Compliance with these brain functions physics is the same for all mammals, so it can be considered a base. This is what allows you to extrapolate the results of the study of the brain of animals on the human brain. However, only a hominid organism (homo erectus) in step bipedalism acquired susceptibility to factor phylogeny geocosmic scale, under whose influence in a social environment in his brain began to take shape and develop structures responsible for speech and thinking. Anatomical differences in the brain of modern humans and apes pronounce in the structure and volume of the frontal-temporal lobes of the neocortex. A key role in the physics of thinking plays a structural and functional asymmetry of the hemispheres of the brain, which is absent in animals and has a racial-sexual differentiation in humans. The genesis of this 
asymmetry could be deterministic physics restructuring of genitals, hands, vision and hearing at the stage of bipedal locomotion and in the development of skills for the conscious work. From these data, based on the cognitive functions of the brain set was physically installed fronto-temporal lobe neocortex and chirality communications brain as hemispheric so and somatic and with the external environment.

The aim is to study the chemical processes in the brain in terms of the magnetic field.

\section{Theory}

Electromagnetic induction is essential to the human nervous system. It is based on communication and signaling functions which are the ability of nerve cells to generate and conduct electrical impulses.

Phase rise ROP lasts about $0.1-0.2 \mathrm{~m} / \mathrm{s}$, and the relaxation time of the membrane potential of the order of $\sim 1 \mathrm{~m} / \mathrm{s}$, and pulse bias currents, corresponding to a phase of growth will be much more than the current relaxation. Changing the charge on the inner side of the membrane of the axon gives rise to a wave of polarization or bias current in the field of myelin paranodal segment. The magnitude of the perturbation will decay exponentially with the distance and speed of the spread will not exceed the speed of the AFP in the nerve (about $1 \mathrm{~m} / \mathrm{s}$ ). The presence of windows due paranodal loops with axoplasm converts wave polarization in the ring current shift in the spirals of loops.

Thus, the generation of ROP in the nodes of Ranvier, associated with the induction of radiation-otshnurovkoy vortex electromagnetic rays, are modeled metric vortices. It is possible that this is the main function of the myelin sheaths of the end coils and spirals incisions. The direction of the vector of energy flux density (Poynting vector) is determined by the sign of the spiral. This factor will provide a one-sided chirality neuron proliferation quantum, and therefore the ROP nerve. Upon reaching the quantum limit with speed $V$ coil myelin segments it can play the role of stimulus to generate ROP next node of Ranvier. This model of a neuron conduction velocity spike will be limited to the excitation current in the coils of the end time of the order of $10^{-6} \mathrm{c}$. The average transfer rate of ROP range from one end to another segment of the myelin in the length of about 100 microns, and determines the speed of saltatory conduction mechanism is $100 \mathrm{~m} / \mathrm{s}$.

\section{Results and Discussions}

Kinematics of electromagnetic induction is subject to the first Maxwell Eq. (1):

$$
\operatorname{rot} \mathrm{E}=-\partial \mathrm{B} / \partial \mathrm{t}
$$

where, $\mathrm{E}$ and $\mathrm{B}$ - mutually orthogonal vector of the vortex electric and magnetic fields.

Using Eq. (1) for the closed loop current is obtained an equation for the self-induction electromotive force (U):

$$
\mathrm{U}=-\mathrm{L}(\mathrm{d} \mathrm{J} / \mathrm{dt})=-\mathrm{d} \Phi / \mathrm{dt}
$$

where, L-inductance of the circuit; J-current, and $\Phi=\mathrm{LJ}$-linkage of self-induction loop.

The effects of electromagnetic induction in a variety of structures and environments of a living organism, with its local magnetic $(\mu)$ and dielectric $(\varepsilon)$ characteristics, subject to the second Maxwell Eq. (2):

$$
\operatorname{rot} \mathrm{H}=\mathrm{j}+\partial \mathrm{D} / \partial \mathrm{t}
$$

where,

$$
\mathrm{D}=\varepsilon_{0} \varepsilon \mathrm{E}, \mathrm{B}=\mu_{0} \mu \mathrm{H}
$$

$j$-bias current and electrodynamic constant vacuum $\left(\varepsilon_{0} \mu_{0}\right)$ and the environment related to the speed of propagation of electromagnetic quantum vacuum $(\mathrm{C})$ and medium $(\mathrm{V})$ relations:

$$
\mathrm{C}=\left(\varepsilon_{0} \mu_{\mathrm{o}}\right)^{-1 / 2}, \mathrm{~V}=\mathrm{C}(\varepsilon \mu)^{-1 / 2}=\mathrm{C} / \mathrm{n}
$$

Extrapolation of the phenomenon of electromagnetic induction can be illustrated by the oscillating circuit.

For a perfect contour of harmonic frequency of electromagnetic waves is given by:

$$
\mathrm{w}=(\mathrm{LC})^{-1 / 2}
$$

Using the value of the potential difference that meets ROP typical neuron $(\mathrm{U} \sim 70 \mathrm{mV})$, the authors 
estimate the amount of electric power used to excite ROP at the nodes of Ranvier saltatory conduction mechanism of the neuron. To represent this interception of a cylindrical capacitor electrode is formed of a neuron membrane and equal in length to intercept (f). The change in energy of the capacitor (W), can be estimated by the formula:

$$
\mathrm{W}=\left(\mathrm{U}^{2} \mathrm{C}\right) / 2
$$

The $C$ for a cylindrical capacitor plate separation (d) and the radius of the inner cylinder (R) under the condition $\mathrm{d} \ll \mathrm{R}$ will equal

$$
\mathrm{C}=\left(2 \pi \varepsilon_{\mathrm{o}} \varepsilon \mathrm{f}\right) /[\ln (1+\mathrm{d} / \mathrm{R})] \approx\left(2 \pi \varepsilon_{\mathrm{o}} \varepsilon \mathrm{fR}\right) / \mathrm{d}
$$

and the value

$$
\mathrm{W}=\left(\mathrm{U}^{2} \pi \varepsilon_{0} \varepsilon \mathrm{fR}\right) / \mathrm{d}
$$

Eq. (8) is the values for the nerve with $\mathrm{R}=5 \mathrm{~mm}: \mathrm{U}$ $\sim 0.07 \mathrm{~V} ; \varepsilon_{\mathrm{o}}=8.8510-12 \mathrm{~F} / \mathrm{m} ; \varepsilon \sim 5 ; \mathrm{f} \sim 10-7 \mathrm{~m} ; \mathrm{d} \sim$ 10-8 $\mathrm{m}$, the authors obtain

$$
\mathrm{W} \sim 5.10^{-17} \mathrm{~J} \text { or } 3.10^{7} \mathrm{~J} / \mathrm{mol}
$$

The same quantity $\mathrm{W}$ is obtained by substituting in Eq. (7) the value of $\mathrm{C}=10^{-2} \mathrm{~F} / \mathrm{m}^{2}$ at the same parameters node of Ranvier and the largest $\mathrm{U}$ at the same parameters node of Ranvier and quantity $U$. The Eq. (9) is comparable to the energy released by the oxidation $\sim 10$ of glucose molecule and hydrolysis $\sim 10^{3}$ molecules of ATP.

It is known that in one molecule of ATP hydrolysis through the membrane tested $\sim 3 \mathrm{Na}^{+}$ions in exchange for $\mathrm{K}^{+}$ion is two, and when ROP excitation flux of $\mathrm{Na}^{+}$ions through the membrane interception is $\mathrm{J} \mathrm{Na} \sim$ $4.10^{3}$ ions $/ \mathrm{mm}^{2}$. Then the number included in the axon of $\mathrm{Na}^{+}$ions is equal $\mathrm{J} \mathrm{Na}(2 \pi \mathrm{Rf}) \sim 10^{4} \sim 3.10^{3}$ it corresponds to ATP, which is the total energy of the order of magnitude is consistent with Eq. (9). When the concentration of ATP in squid axon axoplasm $1 \mathrm{mmol}$ per $1 \mathrm{~kg}$ of $\mathrm{H}_{2} \mathrm{O}$, the total number of molecules of ATP in the cylinder node of Ranvier (radius $5 \mathrm{~mm}$ and a length of $1 \mathrm{~m}$ ) is equal to $\sim 4.10^{7}$ molecules. Consequently, the value $\mathrm{W}$ will be only $0.01 \%$ of the total energy source node of Ranvier.

For the upper limit of energy can take the energy of a photon with a wavelength of $600 \mathrm{~nm}$ (4.10-19 J), which is sufficient to initiate a signal in the receptor cells of the retina.

Energy quasiphotons different types of changes in a wide range, the upper boundary of which can be regarded as energy quasiphoton stimulating the generation of PD in the nodes of Ranvier ( 10-19 J). For the lower limit of the energy quasiphoton can take the energy vortex electromagnetic field generated by eye movement. The density of the energy at $\mathrm{B} \sim 4 \mathrm{pT}$ is of the order:

$\mathrm{E}=\mathrm{B}^{2} /\left(2 \mu_{\mathrm{o}} \mu\right) \sim 10^{-23} \mathrm{~J} / \mathrm{cm}^{3}$, or about $0.01 \mathrm{~kJ} / \mathrm{mol} \mathrm{cm}{ }^{3}$.

The degree of influence of the magnetic field on the sensitive micro and macro structure of the brain and the environment will be determined by the magnitude of the energy density:

$$
\mathrm{E}=(\mathrm{M} \mathrm{B}) / 2,
$$

where, $M$ - specific magnetization (specific gravity of the magnetic moments - $\mathrm{m}$ ), equal to:

$$
\mathrm{M}=\Sigma \mathrm{m} \text {. }
$$

In the case of circular currents $(\mathrm{J}) \mathrm{m}=\mathrm{J} \Delta S, \Delta S$ where in the surface area covered by the current. The polarizing effect of the magnetic field can be amplified under the influence of the thermal motion of particles in the medium.

Summary energy effect all stages of enzymatic reaction of glucose oxidation in mitochondria has its limit thermal effect of the combustion reaction in a oxygen atmosphere glucose:

$$
\mathrm{C}_{6} \mathrm{H}_{12} \mathrm{O}_{6}+6 \mathrm{O}_{2} \rightarrow 6 \mathrm{CO}_{2}+6 \mathrm{H}_{2} \mathrm{O}+2800(\mathrm{~kJ} / \mathrm{mol}) .
$$

The reaction of glucose oxidation in mitochondria involves reacting ATP synthesis, wherein one molecule of glucose with 38 molecules of ATP. When an anaerobic glycolysis of glucose produced only two ATP molecules and two lactate molecules are chiral, which apparently contribute chirality eye and brain nutrition. The maximum yield of metabolic energy gives 38 ATP hydrolysis schemes:

$$
\begin{gathered}
\mathrm{AТФ} \rightarrow \text { АДФ + } \sim \mathrm{P}+36(\mathrm{~kJ} / \mathrm{mol}) \\
\mathrm{P} \sim \mathrm{P} \rightarrow \mathrm{P}+\mathrm{P}+33,4(\mathrm{~kJ} / \mathrm{mol})
\end{gathered}
$$


The total energy of 38 high-energy bonds of ATP molecules is $2,640 \mathrm{~kJ} / \mathrm{mol}$, which is $\sim 95 \%$ of the limit values of the energy of combustion of one molecule of glucose. This shows the high efficiency of transformation of the enzymatic reactions quasiphotons corresponding $\sigma-\mathrm{C}-\mathrm{C}$ bonds, $\mathrm{C}-\mathrm{O}-\mathrm{C}$, $\mathrm{C}-\mathrm{H}$ glucose quasiphotons localized on the two high-energy $\mathrm{P}=\mathrm{O}$ bonds in ATP.

Assume that quasiphotons equiprobably distributed relations reaction products of glucose oxidation and hydrolysis of ATP, then the limit values quasiphotons energies that meet these reactions will be equal to $1 / 12$ and $1 / 152$ of the heat of Eq. (12) equal to $4.5 \cdot 10^{-18} \mathrm{~J}$, i.e. $\sim 3 \sim 3 \cdot 10^{-19}$ and $10^{-20} \mathrm{~J}$, respectively. If these quasiphotons apply the universal relationship between energy and the characteristic size (r) of the discrete elements of matter ( $v / g$-couple elementary particle):

$$
\mathrm{E} \sim \hbar \mathrm{c} / \mathrm{r}
$$

then quasiphoton acting as a quantum of metabolic energy, the authors obtain the radius of $\sim 1$ micron, which is comparable to the radius of the axon.

It can be imagined that quasiphotons participate in the DNA double helix unwound as follows. In the replication fork sugar-phosphate backbone of the DNA chain resonantly absorb metabolic quasiphotons oscillatory type. It increases the elasticity of the chains, which leads to the rupture of hydrogen bonds between them. Given that the two sugar-phosphate DNA helix has to link one hydrogen bond and its energy is equal to $\sim 19 \mathrm{~kJ} / \mathrm{mol}\left(3 \cdot 10^{-20} \mathrm{~J}\right)$, the authors find that the gap is sufficient for its absorption of the DNA chain of metabolic quasiphoton.

Presence isomorphic amino acid fragments in the peptide chains of protein structure and neurotransmitters (glycine, acetylcholine, glutamic acid, dopamine, serotonin) allows to offer the transmission mechanism quasiphoton resonant oscillatory type in contact with a neurotransmitter receptor. Due to the presence in the structures of electron-mediators, proton-donor and acceptor groups of the electronic ground state is characterized by intramolecular charge transfer $Д^{+\delta}-\mathrm{C}-\mathrm{A}^{-\delta}$. Where D - with an amino group donor, a methoxy group, a benzene ring, and A-acceptor carbonyl and hydroxyl groups, and $\mathrm{C}$ - chain of $\sigma$-bonds. This factor and susceptibility of mediators of forming hydrogen bonds are the basis of their physical and chemical sorption to the receptors of the postsynaptic membrane. The receptor, accepting or giving quasiphoton in contact with neurotransmitter changes its conformation, locking or opening the calcium channel membrane.

\subsection{Thermodynamics of the Brain}

Brain can be generally regarded as the reaction thermodynamic system occurring in steady-state. The influx of energy and discharge of excess heat in the brain are balanced a narrow range of temperatures from $\sim 37{ }^{\circ} \mathrm{C}$ (center of the brain) to $\sim 36{ }^{\circ} \mathrm{C}$ (the cerebral cortex). This temperature gradient being due to the lower temperature of the environment can play a significant role in guiding the heat flows within the brain. Features of the thermodynamics of phase transitions formed the basis of the mechanism of adaptation of living systems, which, in fact, is constant-energy transitions and transitions with the activation energy of the order $\mathrm{k} \Delta \mathrm{T}$ (at $\Delta \mathrm{T} \sim 0,1-1 \mathrm{~K}$ ) between the states of varying degrees of order or similar protein molecules, molecular-kletochnyh ensembles. Reducing the entropy component of the internal energy of a living system involves resonant absorption of a quantum of its external or em-neutrino energy that it converts the active metabolic quasiphoton.

Thus, the brain combines thermodynamics of equilibrium thermodynamics, metabolism and stationary nonequilibrium thermodynamics neural network, "working body" which is a Bose gas quasiphoton. Accordingly, the internal energy $U$ of the brain as a function of its state depends in general on the temperature (or entropy $\mathrm{S}$ ), from the strain tensor $G$, which depends on the intracranial pressure, the magnetic moment $\mathrm{M}$ of individual metabolites and 
macrostructures of the total angular momentum of the nuclei and atoms $\mathrm{L}$ and on the polarization of the medium P. Consequently, the total differential of the internal energy $\mathrm{U}=\mathrm{U}(\mathrm{S}, \mathrm{G}, \mathrm{M}, \mathrm{L}, \mathrm{P})$ has the form:

$$
\mathrm{d} U \equiv \mathrm{TdS}-\Pi \mathrm{dG}+\mathrm{BdM}+\mathrm{DdP}+\mathrm{FdJ}
$$

where, $T$ - the absolute temperature of the system; $\Pi$-pressure tensor; $F$-vector orientation of the polarization of the spin or angular momentum. In the Eq. (13) member FdL characterizes the work related to the orientation of the polarization of nuclear spin, or angular momentum of atoms and molecules (the same way as members of DdP and BdM define work related to polarization and magnetization of the system).

Orientation and polarization effects play an essential role in the initiation of phase transitions in homogeneous systems of the brain and the body.

One way to metabolize energy neutrino is the reaction of synthesis in the neurohormone melatonin pineal gland, which plays a key role in the process of sexual maturation and mental retardation.

The frequency spectrum of rhythmic electrical activity of the brain is responsible in the hierarchy of the various structures of the brain. By the steady rhythm EEG include: delta rhythm $(0.5-4 \mathrm{~Hz})$; theta rhythm (5-7 Hz); alpha-rhythm (8-13 Hz); mu wave - on the frequency amplitude characteristics similar to the alpha rhythm, but is prevalent in the anterior regions of the cerebral cortex; beta rhythm $(15-35 \mathrm{~Hz})$ and gamma wave (above $35 \mathrm{~Hz}) . \mathrm{Mu}$ rhythm, is possibly connected with the electromagnetic activity of the eyes and a rhythmic scanning EM swirl frontal lobes. When you remove the thalamus or cut off its links with bark alpha rhythm disappears.

The currents in the nerve structures and the reticular formation of the medulla can generate eddy magnetic fields in the structures of the pons and cerebellum.

Time mastering one bit of visual information is $15-50 \mathrm{~m} / \mathrm{s}$. Time elemental act of thinking is in the range of $150-300 \mathrm{~m} / \mathrm{s}$. In awareness of the meaning of a single word it takes an average of about $200 \mathrm{~m} / \mathrm{s}$.
This time constant can be associated with theta rhythm, which manifests cortico-limbic interactions regulating emotions and mental activity. Since the typical transmission times ORP within the structures of the brain of the order of no more than about $10 \mathrm{~m} / \mathrm{s}$, it is possible to assume that the act of thinking is limited by the speed of the chemical mechanism of encoding information that requires the activation of synaptic connections.

\subsection{Consciousness and Memory}

Chemistry of consciousness or self-awareness, being identical to the physics of thought and speech, based on the brain's ability to respond to external signals, converting them into electromagnetic neural network codes, memorize and learn the codes, transform and synthesize them into a matrix of thought forms. All these operations are possible if the brain physicochemical mechanisms of activation and reading the memory-preserving codes. The process of reading and recognition of the thought-form implies the presence of a stable dynamic reference system code-frame constituting the physical basis of consciousness of the subject. This system is formed in the course of phylogeny and ontogeny of the genetic program in the continuous exposure to the brain through the senses (sight, hearing, touch, smell, taste) of the whole range of physical and social organizing factors.

The gene codes, make the baseline memory function throughout a person's life, defining racial, sexual and mental features of its physical brain. The next level of memory is unconditioned reflexes, the principles of its organization laid at the stage of morphogenesis. At this stage it creates a chemical basis of memory resource initial sensory impressions and sensations.

First, the gene level memory implemented spontaneously on the molecular level in the synthesis of cellular nuclear DNA, which in turn, is programmed growth and function of the nervous cells. 
Thermodynamics of the tertiary structure of protein molecules (DNA, hemoglobin, enzymes) allow them to condense the heat or metabolic quasiphotons, transforming them into energy vibrational-rotational motion of atoms characteristic amino acid groups. Upon reaching the threshold excitation energy is transferred to it by fractal-resonance mechanism on the metabolite, thereby initiating its participation in the chemical synthesis of well-defined structure. So it may be broadcasting genetic information from the molecular to the macroscopic level of the organization of living systems.

The following levels of memory are formed by stable development of neural connections between areas of the cortex and the basal ganglia, the limbic brain, the thalamus, and cerebellum.

\section{Conclusions}

A study of the influence of magnetic field on the brain, the authors examined the redox potentials and the thermodynamics of the brain.

Chemistry of the human brain has two components - the basic chemistry common to all mammals and chemistry of thinking inherent to man. The authors proposed a mechanism of induction and thermodynamic features of the brain. The authors offered the mechanisms of the work RAM (random-access memory) (physical) and permanent (chemical), the brain's memory, including the model of nonlocal quantum correlations. Chemical thinking integrates to some extent a function of all the structures of the brain. Limiting mental reaction time or the act of thinking is of the order of $\sim 100 \mathrm{~m} / \mathrm{s}$.
During this time in the brain are utilized by tens of thousands of synaptic connections are activated, and dozens of cortical areas and subcortical structures. Even if the parallel operations in neural networks and high-speed reflexive and associative links between the areas of the cortex and memory blocks for targeting functional activity of the brain it should work the quantum mechanism of search and selection of information, the kinetics of which is not to be limited to the rate of transfer of matter and energy.

\section{References}

[1] Agranoff, B. W. 2003. "History of Neurochemistry." Encyclopedia of Life Sciences.

[2] Siegel, G. J., Albers, R. W., Brady, S. T., and Price, D. L. 2006. Basic Neurochemistr. 7th ed. Academic Press.

[3] Brown, D. R. 2002. Prion Diseases and Copper Metabolism. Chichester: Horwood Press.

[4] Kozlowski, H., Brown, D. R., and Valensin G. 2006. Metallochemistry of Neurodegeneration. UK: Royal Society of Chemistry Publishing.

[5] Nutt, D., Baldwin, D., and Aitchison, K. 2013. "Benzodiazepines: Risks and Benefits: A Reconsideration.” J. Psychopharmacol 27 (11): 24.

[6] Candace P. 1999. "Molecules of Emotion." The Science between Mind-Body Medicine Scribner: 236.

[7] Quastel, J. H, and Wooldridge, W. R. 1928. "Some Properties of the Dehydrogenating Enzymes of Bacteria." The Biochemical Journal 22 (3): 689-702.

[8] Smythies, J. R., and Charles, C. T. 1963. Schizophrenia: Chemistry, Metabolism and Treatment. Springfield, Illinois.

[9] Tilli, T. 2012. "The History of Modern Biomedicine Research Group.” Queen Mary, University of London.

[10] Thudichum, J. 1884. A Treatise on the Chemical Constitution of the Brain.

[11] Kholmanskikh, A. 2006. "Physics Modeling of the Brain." Quantum Magic 3 (3): 3126. 